\title{
miR-650 promotes non-small cell lung cancer cell proliferation and invasion by targeting ING4 through Wnt-1/ $\beta$-catenin pathway
}

\author{
XIANGQIN TANG ${ }^{1}$, YANJUN DING $^{2}$, XIAOQING WANG $^{3}$, XIUZHEN WANG $^{4}$, LIN ZHAO $^{5}$ and $\mathrm{HONGMEI} \mathrm{BI}^{6}$ \\ ${ }^{1}$ Department of Oncology, Jining No. 1 People's Hospital, Jining, Shandong 272011; ${ }^{2}$ Department of General Surgery, \\ People's Hospital of Chiping, Chiping, Shandong 252100; ${ }^{3}$ First Ward, Department of Neurology, The Affiliated \\ Central Hospital of Qingdao University, Qingdao, Shandong 266000; ${ }^{4}$ Department of Clinical Nutrition, \\ People's Hospital of Zhangqiu Area, Jinan, Shandong 250200; ${ }^{5}$ Department of Respiratory Medicine, \\ People's Hospital of Rizhao, Rizhao, Shandong 276826; ${ }^{6}$ Department of Respiratory Medicine, \\ The Third People's Hospital of Qingdao, Qingdao, Shandong 266041, P.R. China
}

Received June 4, 2019; Accepted July 30, 2019

DOI: $10.3892 / \mathrm{ol} .2019 .10805$

\begin{abstract}
Non-small cell lung cancer (NSCLC) is the most frequent cancer worldwide with a poor 5-year survival. miR-650 acts as an oncogene and regulates tumor progress in various cancers. Molecular mechanisms of miR-650 in NSCLC cell proliferation and invasion was studied. The mRNA levels of miR-650 and special genes were calculated using RT-qPCR. MTT and transwell assays were applied to measure the proliferative and invasive ability. Kaplan-Meier method was used to assess the survival of NSCLC patients. miR-650 was upregulated in NSCLC and upregulation of miR-650 was associated with a poor overall survival of NSCLC, while the results of ING4 demonstrated the opposite results. miR-650 promoted proliferation and invasion through Wnt- $1 / \beta$-catenin pathway by targeting inhibitor of growth 4 (ING4) in A549 cells. ING4 was a direct target gene of miR-650 and the expression of ING4 was mediated by exogenous altering the expression of miR-650. Remarkably, alterations of ING4 expression eliminated the functions of miR-650 on the proliferation and metastasis of NSCLC. miR-650 enhanced A549 cell proliferation and invasion through Wnt- $1 / \beta$-catenin pathway by directly targeting the 3'-UTR of ING4 mRNA. The newly identified miR-650/ING4 axis provides a novel insight into the pathogenesis of NSCLC.
\end{abstract}

\section{Introduction}

Non-small cell lung cancer (NSCLC) accounts for approximately $80 \%$ of lung cancer and is the leading cause of cancer-related death worldwide (1). Despite great advances in

Correspondence to: Dr Hongmei Bi, Department of Respiratory Medicine, The Third People's Hospital of Qingdao, 29 Yongping Road, Qingdao, Shandong 266041, P.R. China

E-mail: qsz4781195216p@163.com

Key words: miR-650, inhibitor of growth 4, metastasis, non-small cell lung cancer, upregulation, prognosis therapeutic methods, the 5-year overall survival of NSCLC is still $<15 \%$ (2). Therefore, it is urgent to explore novel biomarkers for disease prevention and clinical treatment of NSCLC.

MicroRNAs (miRNAs) are a class of endogenous, non-coding small RNAs with 19-25 nucleotides in length $(3,4)$. miRNAs binding to the 3'-UTR of target mRNA to incise the target mRNAs or inhibit the translation of proteins at post-transcriptional level (3). Accumulating evidence has identified that miRNAs participate in multiple processes of cancer, including cell growth, metastasis and apoptosis (5). miR-650 functions as an oncogene and is overexpressed in multiple diseases, including colorectal cancer, chronic lymphocytic leukemia, osteosarcoma and glioma (6-9). Mraz et al indicated that the expression of miR-650 was high and affected the biology of chronic lymphocytic leukemia (10). Moreover, miR-650 affected the release of allograft rejection-associated cytokines from HRGECs and regulated chemotaxis of macrophages (11). However, miR-650 inhibited the proliferation and metastasis through targeting to Gfil in oral cancer and leukemia $(12,13)$. Therefore, we investigated miR-650 expression and the roles of miR-650 in NSCLC.

Inhibitor of growth 4 (ING4) is a member of IMG family, which is involved in nucleoprotein modification by acetylation of histone $(14,15)$. ING4 containing a highly conserved C-terminal plant homeodomain finger motif, may regulate several biological activities, including DNA repair, cell cycle and apoptosis (16). Moreover, ING4 has been reported to be a tumor suppressor gene in various cancers, including head and neck squamous cell carcinomas, pancreatic cancer, breast cancer and clear cell renal carcinoma (17-21). Downregulation of ING4 improved angiogenesis of transformed gastric epithelial cells (22). ING4 inhibited cell proliferation, cell cycle progress, migration and invasion in melanoma (23). Moreover, Chen et al demonstrated that ING4 suppressed tumor angiogenesis and acted as a prognostic marker in human colorectal cancer (24). In lung cancer, ING4 suppressed the proliferation and increased apoptosis, and ING4 overexpression enhanced radiosensitivity (25). In this study, miR-650 was validated to be upregulated in NSCLC and upregulation of miR-650 improved 
the overall survival of NSCLC, while ING4 demonstrated the opposite results. miR-650 promoted cell proliferation and invasion and ING4 could partially reverse the function of miR-650. ING4 was confirmed as a direct and functional target of miR-650, and miR-650 enhanced Wnt-1/ $\beta$-catenin pathway in A549 cells.

\section{Patients and methods}

Tissue samples. Forty-nine NSCLC patients were collected at Jining No. 1 People's Hospital (Jining, China) from January 2015 to June 2018 and 49 pairs of cancer tissues and adjacent normal tissues were obtained. The tissue specimens were immediately frozen in liquid nitrogen and stored at $-80^{\circ} \mathrm{C}$. None of the patients accepted any treatment prior to surgery and the patient details are shown in Table I.

The Ethics Committee of Jining No. 1 People's Hospital approved this study. Patients who participated in this research had complete clinical data. The signed informed consents were obtained from the patients or the guardians.

Cell lines and culture condition. Two human lung cell lines, A549 (cat. no. CCL-185) a lung adenocarcinoma and NCI-H460 (cat. no. HTB-177) a large cell carcinoma and one normal bronchial epithelial cell line MRC-5 (cat. no. CCL-171) were purchased from American Type Culture Collection. RPMI-1640 medium (Gibco; Thermo Fisher Scientific, Inc.) supplemented with 10\% FBS (Gibco; Thermo Fisher Scientific, Inc.) was utilized to culture all the cells at $37^{\circ} \mathrm{C}$ in a humidified atmosphere of $5 \% \mathrm{CO}_{2}$.

Cell transfection. The miR-650 mimic, the miR-650 inhibitor and negative control (NC) were designed and synthesized by GenePharma, while pcDNA3.1-ING4 and pcDNA3.1-NC vectors were purchased from Guangzhou RiboBio Co., Ltd. A549 cells at 70\% confluence in 6-well plates were transfected with the vectors by Lipofectamine 2000 (Invitrogen; Thermo Fisher Scientific, Inc.). After incubated for $6 \mathrm{~h}$ at $37^{\circ} \mathrm{C}$ in a humidified atmosphere of $5 \% \mathrm{CO}_{2}$, the cells were replaced with fresh normal RPMI-1640 medium in each well.

Total RNA extraction and RT-qPCR. The TRIzol reagent (Invitrogen; Thermo Fisher Scientific, Inc.) was applied to extract total RNA from tissue samples and cell lines. For the quantification of ING4 mRNA, the PrimeScript RT reagent kit (Takara Bio) was utilized to perform reverse transcription. The temperature conditions for reverse transcription were as follows: $37^{\circ} \mathrm{C}$ for $15 \mathrm{~min}$ and $85^{\circ} \mathrm{C}$ for $5 \mathrm{sec}$. SYBR Premix Ex Taq (Takara Bio, Inc.) was applied to carry out qPCR on Applied Biosystems 7500 Real-time PCR System (Applied Biosystems; Thermo Fisher Scientific, Inc.). The expression of miR-650 was determined by using TaqMan microRNA assay (Applied Biosystems; Thermo Fisher Scientific, Inc.). GAPDH and U6 served as internal control for ING4 and miR-650 respectively. PCR conditions were as follows: 1 cycle of $95^{\circ} \mathrm{C}$ for $30 \mathrm{sec}$, followed by 40 cycles of a two-step cycling program $\left(95^{\circ} \mathrm{C}\right.$ for $5 \mathrm{sec} ; 60^{\circ} \mathrm{C}$ for $\left.30 \mathrm{sec}\right)$. PCR primer sequences were as follows: miR-650 forward 5'-AGAGGAGGCAGCGC TCT-3' and reverse 5'-CAGTGCGTGTCGTGGAGT-3'; U6 forward, 5'-GCTTCGGCAGCACATATACTAAAAT-3', reverse, 5'-CGCTTCACGAATTTGCGTGTCAT-3'; ING4 forward 5'-TTTCAGAGGGAGGGTCCTTT-3' and reverse 5'-GCCAGAGCCTAGATGACCTG-3'; GAPDH forward, 5'-CTGGGCTACACTGAGCACC-3', reverse, 5'-AAGTGG TCGTTGAGGGCAATG-3'. The $2^{-\triangle \Delta C q}$ method was applied to assess the relative expression of the mRNA levels of miR-650 and ING4 (26).

Western blot analysis. RIPA containing protease inhibitors (Beyotime Institute of Biotechnology) was adopted to extract the total proteins on ice. Total protein concentration was quantified using BCA assay kit (Beyotime Biotechnology), equal amounts of proteins $(50 \mu \mathrm{g})$ were separated by $10 \%$ SDS-PAGE, and transferred onto PVDF membranes (EMD Millipore). Then the membranes were blocked in 5\% non-fat milk to block the non-specific antigen, and then the primary antibodies were incubated at $4^{\circ} \mathrm{C}$ at room temperature for $1.5 \mathrm{~h}$ overnight with anti-ING4 antibody (cat. no. ab113425; dil 1:1,000), anti-Wnt1 (cat. no. ab15251; dil 1:1,000) anti- $\beta$-catenin (cat. no. ab16051; dil 1:1,000) and anti-GADPH antibody (cat. no. ab125247; dil 1:3,000) all from Abcam. After the membranes were washed with TBST, the corresponding secondary antibody was incubated with HRP-conjugated (dil 1:4,000; Abcam) at room temperature for $2 \mathrm{~h}$. Enhanced chemiluminescence solution (Pierce; Thermo Fisher Scientific, Inc.) was adopted to visualize the protein bands and then were analyzed with AlphaEase FC 4.0.1 software (ProteinSimple).

CCK8 assay. CCK8 (Dojindo) was employed to calculate the capacity of cell proliferation. A549 cells at density 3,000 cells/ well, were seeded into 96-well plates and cultured for 24, 48, 72 or $96 \mathrm{~h}$ at $37^{\circ} \mathrm{C}$. Subsequently, each well was added with $10 \mu \mathrm{l}$ CCK 8 reagent and incubated $2 \mathrm{~h}$. Finally, microplate reader (Bio-Rad Laboratories) was employed to determine cell proliferation with the absorbance at $450 \mathrm{~nm}$.

Transwell assays. Transwell chambers $(8 \mu \mathrm{M}$ pore size, Corning Inc.) covered with Matrigel (BD Biosciences) were applied to measure the capacity of cell invasion. A549 cells were suspended in normal medium without FBS, and $200 \mu \mathrm{l}$ were seeded into the upper chamber, while the lower chambers were filled with $500 \mu 1$ RPMI-1640 medium supplemented with $20 \%$ FBS functioned as the attractant. After incubation for $48 \mathrm{~h}$, the cells remaining on the upper surface of the membranes were removed by cotton swabs, whereas the invaded cells were fixed and stained by paraformaldehyde and $10 \%$ crystal violet (Sigma-Aldrich; Merck Millipore) respectively. Microscopy (Olympus Corporation; magnification, x200) was employed to count the invaded cell numbers in five fields.

Luciferase reporter assay. To verify the putative targets of miR-650, we adopted bioinformatics tool TargetScan (http:// targetscan.org/) to predict the candidate genes. The binding site at mRNA 3'-UTR was mutated from UGCCUCC to ACGGAGG, and then inserted into pmirGlo vectors, which were designated as pmirGlo-ING4-WT and pmirGlo-ING4MUT. Both the wild-type and the mutant sequences and miR-650 mimic were co-transfected in A549 cells. Dual Luciferase Assay System (Promega Corporation) was employed 
Table I. miR-650 expression and clinicopathological features in 49 non-small cell lung cancer.

\begin{tabular}{|c|c|c|c|c|}
\hline \multirow{2}{*}{$\begin{array}{l}\text { Clinicopathological } \\
\text { features }\end{array}$} & \multirow[b]{2}{*}{ Cases $(n=49)$} & \multicolumn{2}{|c|}{ miR-650 expression } & \multirow[b]{2}{*}{ P-value } \\
\hline & & $26 \operatorname{High}(\%)$ & 23 Low $(\%)$ & \\
\hline Sex & & & & 0.469 \\
\hline Male & 25 & $12(48.0)$ & $13(52.0)$ & \\
\hline Female & 24 & $14(58.3)$ & $10(41.7)$ & \\
\hline Age (years) & & & & 0.648 \\
\hline$\leq 60$ & 23 & $13(56.5)$ & $10(43.5)$ & \\
\hline$>60$ & 26 & $13(50.0)$ & $13(50.0)$ & \\
\hline Tumor size (mm) & & & & 0.124 \\
\hline$\leq 5.0$ & 27 & $17(63.0)$ & $10(37.0)$ & \\
\hline$>5.0$ & 22 & $9(40.9)$ & $13(59.1)$ & \\
\hline TNM stage & & & & $0.016^{\mathrm{a}}$ \\
\hline I-II & 26 & $18(69.2)$ & $8(30.8)$ & \\
\hline III-IV & 23 & $8(34.8)$ & $15(65.2)$ & \\
\hline Local invasion & & & & 0.066 \\
\hline $\mathrm{T} 1-\mathrm{T} 2$ & 26 & $17(65.4)$ & $9(34.6)$ & \\
\hline $\mathrm{T} 3-\mathrm{T} 4$ & 23 & $9(39.1)$ & $14(60.9)$ & \\
\hline Lymph node metastasis & & & & $0.029^{\mathrm{a}}$ \\
\hline $0-2$ & 23 & $16(69.6)$ & $7(30.4)$ & \\
\hline$>2$ & 26 & $10(38.5)$ & $16(61.5)$ & \\
\hline Histology & & & & 0.765 \\
\hline Adenocarcinoma & 33 & $18(54.5)$ & $15(45.5)$ & \\
\hline Squamous & 16 & $8(50.0)$ & $8(50.0)$ & \\
\hline
\end{tabular}

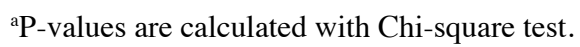

to calculate the firefly luciferase activity with renilla luciferase activity as the normalization.

Statistical analysis. SPSS 19.0 was used to analyze all the data and presented as mean \pm standard deviation (SD). The one way analysis of variance followed by Tukey's post-hoc test and student's t-test (for comparisons between two groups) were employed to perform the statistical analysis. The relationship between expression of miR-650 and clinical and the pathological variables was analyzed using Pearson's $\chi^{2}$ test. $\mathrm{P}<0.05$ was considered to indicate a statistically significant difference.

\section{Results}

Overexpression of miR-650 is associated with poor prognosis. miR-650 expression in NSCLC tissues and their matched adjacent normal tissues was calculated by RT-qPCR. The mRNA levels of miR-650 were lower in normal lung tissues than that in NSCLC tissues $(\mathrm{P}<0.0001)$ (Fig. 1A). To verify the connection between the expression of miR-650 and overall survival of NSCLC patients, the survival curves were plotted. As shown in Fig. 1B, patients with higher expression of miR-650 had a shorter OS than those with lower expression $(\mathrm{P}=0.0227)$. To investigate the relationship between miR-650 and NSCLC clinicopathological characteristics, 49 patients were divided into two groups according to miR-650 expression, sex, age, tumor size, TNM stage, local invasion, lymph node metastasis and histology. Statistical differences were detected by chi-square test and it was found that miR-650 was associated with tumor size $(\mathrm{P}=0.016)$ and lymph node metastasis $(\mathrm{P}=0.029)$ (Table $\mathrm{I})$.

miR-650 enhances cell proliferation and invasion in NSCLC. Expression of miR-650 was calculated in NSCLC cell lines (A549 and NCI-H460) and a bronchial epithelial cell line (MRC-5). Similar to results in tissues, miR-650 was overexpressed in NSCLC A549 cells $(\mathrm{P}=0.0004)$ and NCI-H460 $(\mathrm{P}=0.0014)$ versus that in normal MRC-5 cells (Fig. 2A), which suggested that miR-650 may play important roles in growth and metastasis of NSCLC. The miR-650 mimic or miR-650 inhibitor as well as the negative control (NC) was transfected with A549 cells. We measured the expression of miR- 650 by RT-qPCR, and it demonstrated that miR-650 was upregulated in A549 cells transfected miR-650 mimic $(\mathrm{P}<0.0001)$, while downregulated after transfected with miR-650 inhibitor $(\mathrm{P}=0.0013)$ (Fig. 2B). Subsequently, CCK8 and Transwell assays were performed to calculate the proliferative and invasive abilities of miR-650. As expected, the cell proliferation $(\mathrm{P}=0.0008)$ and invasion $(\mathrm{P}=0.0004)$ were enhanced by miR-650 mimic, whereas, miR-650 reduced the proliferative $(\mathrm{P}=0.0019)$ and invasive $(\mathrm{P}=0.0044)$ capacities in A549 cells (Fig. 2C and D), which illustrated that miR-650 acted as an oncogene in NSCLC. 

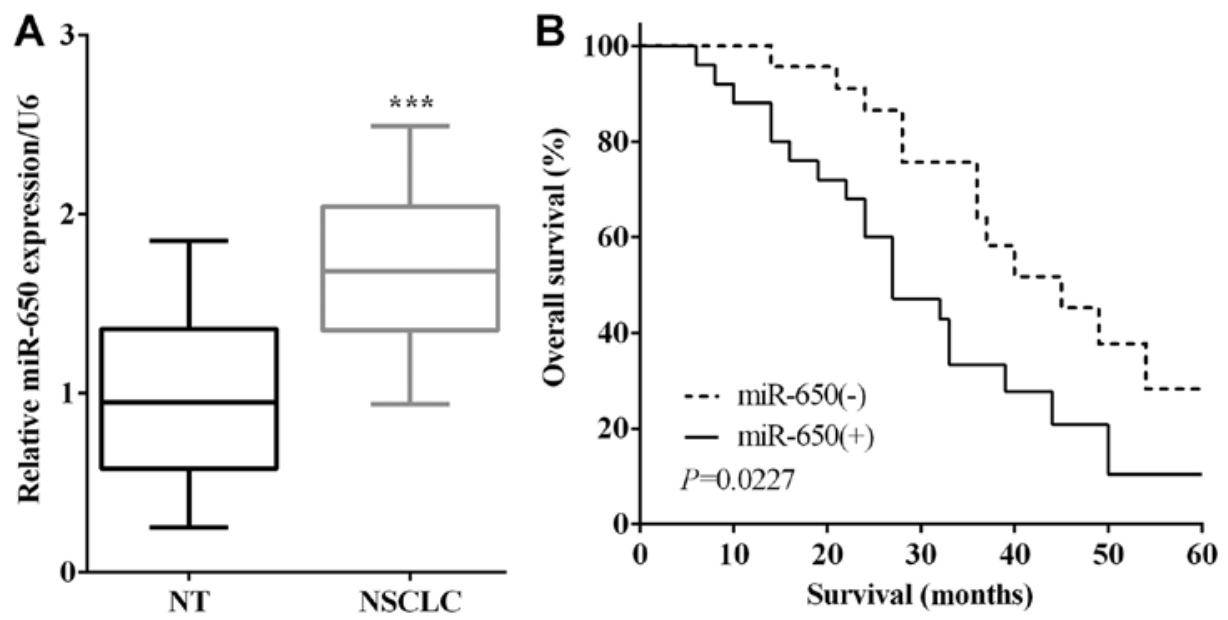

Figure 1. Overexpression of miR-650 is associated with poor prognosis. (A) The expression levels of miR-650 were lower in normal lung tissues than in NSCLC tissues. (B) Patients with higher expression of miR-650 had a shorter OS than those with lower expression. NSCLC, non-small cell lung cancer; NT, normal tissue. ${ }^{* * *} \mathrm{P}<0.001$.
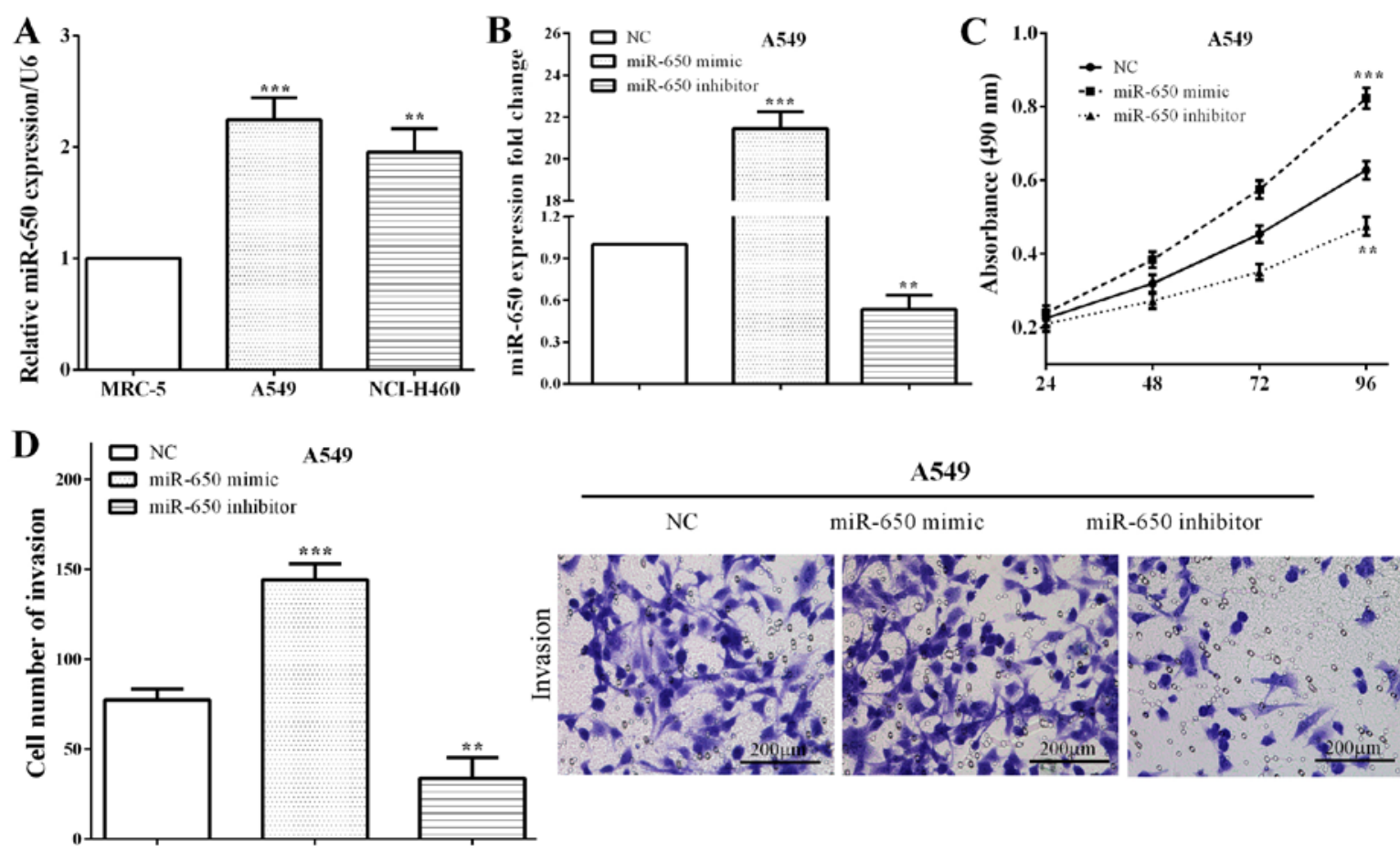

Figure 2. miR-650 enhances cell proliferation and invasion in NSCLC. (A) miR-650 was overexpressed in NSCLC cell lines A549 and NCI-H460 versus that in normal MRC-5 cells. (B) miR-650 was upregulated in A549 cells following transfection of miR-650 mimic, while it was downregulated after transfected with miR-650 inhibitor. (C and D) the cell proliferation and invasion were enhanced by miR-650 mimic, whereas, miR-650 reduced the proliferative and invasive capacities in A549 cells. NSCLC, non-small cell lung cancer; NC, negative control. ${ }^{* *} \mathrm{P}<0.01 ;{ }^{* * *} \mathrm{P}<0.001$.

ING4 is a target gene of miR-650. TargetScan was adopted to explore the potential targets of miR-650, and ING4 was identified (Fig. 3A). To evaluate whether miR-650 directly targeted the 3'-UTR of ING4 mRNA, luciferase reporter assay was performed. A549 cells were co-transfected with the miR-650 mimic and pmirGlo-ING4-WT or pmirGlo-ING4-MUT. As expected, we discovered that the miR-650 mimic reduced the luciferase activity of pmirGlo-ING4-WT $(\mathrm{P}=0.0010)$, whereas the activity of pmirGlo-ING4-MUT was not altered $(\mathrm{P}=0.8645)$ (Fig. 3B). To verify that the expression of ING4 was mediated by miR-650, RT-qPCR was applied to assess
ING4 expression in A549 and NCI-H460 cells when exogenously altered by miR-650. It was shown that miR-650 mimic decreased ING4 expression $(\mathrm{P}=0.0024$ and 0.0364$)$ while miR-650 inhibitor enhanced the expression of ING4 in A549 and NCI-H460 cells ( $\mathrm{P}=0.0007$ and 0.0044) (Fig. 3C).

miR-650 regulates NSCLC progress through $I N G 4 / W n t 1 / \beta$ catenin signaling pathway. ING4 expression was measured by RT-qPCR in cancer and adjacent normal lung tissues, and it was demonstrated that ING4 expession was low in NSCLC tissues versus adjacent normal lung tissues $(\mathrm{P}<0.0001)$ (Fig. 4A). 
A

Site: $261-268$ of ING4

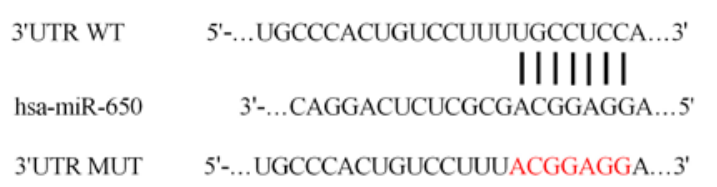
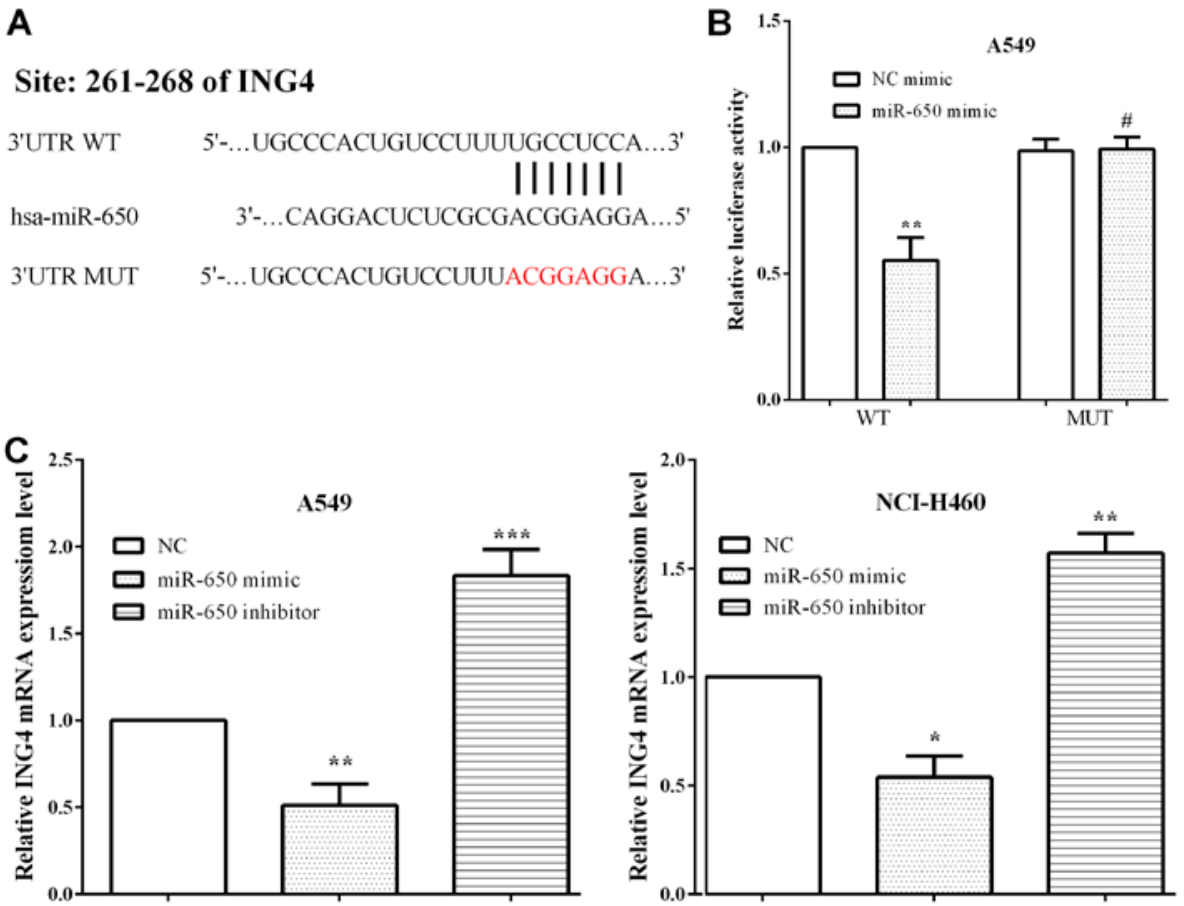

Figure 3. ING4 is a target gene of miR-650. (A) TargetScan predicted that miR-650 targeted the 3'-UTR of ING4 mRNA. (B) miR-650 mimic reduced luciferase activity of pmirGlo-ING4-WT, whereas, the activity of pmirGlo-ING4-MUT was not altered. (C) The mRNA level of ING4 was improved by miR650 mimic while it was decreased by miR-650 inhibitor in A549 and NCI-H460 cells. ING4, inhibitor of growth 4; NC, negative control. ${ }^{*}<0.05 ;{ }^{* * *} \mathrm{P}<0.01$; ${ }^{* * * *} \mathrm{P}<0.001 ;{ }^{*} \mathrm{P}>0.05$.

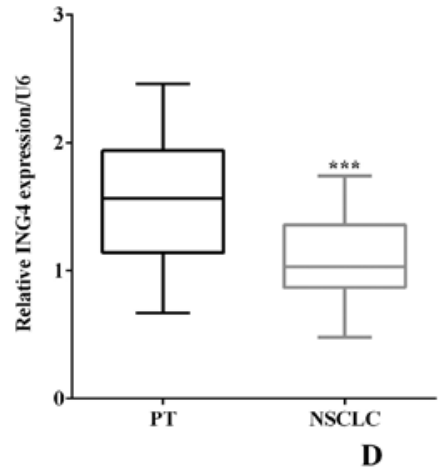

C

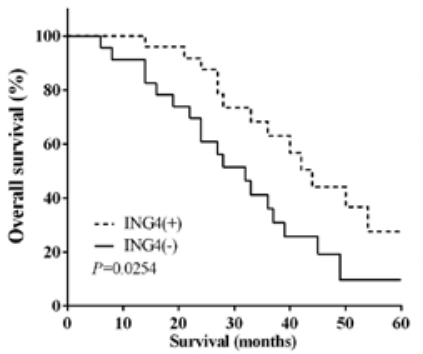

B
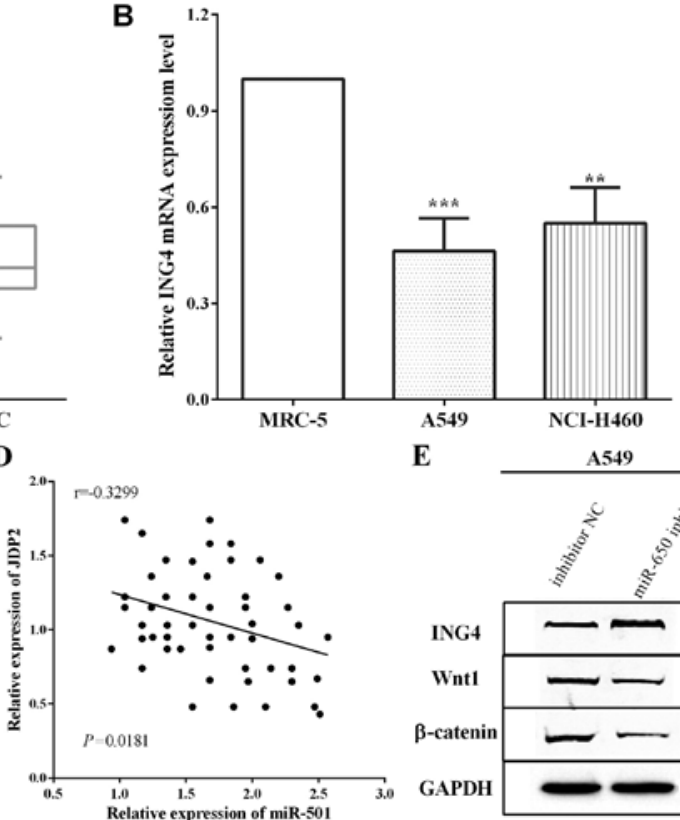

$\mathbf{E}$ A549

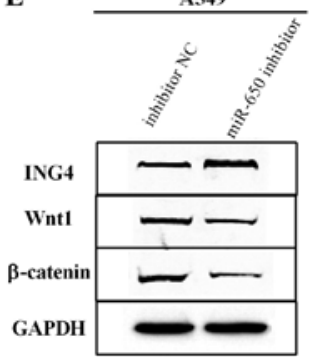

Figure 4. miR-650 regulates NSCLC progress through ING4/Wnt1/ $\beta$-catenin signaling pathway. (A) ING4 expression was low in NSCLC tissues compared with the adjacent normal lung tissues. (B) Downregulation of ING4 predicts poor prognosis. (C) ING4 is downregulated in A549 and NCI-H460 cells compared with that in MRC-5 cells. (D) miR-650 expression has a negative connection with ING4 expression in NSCLC tissues. (E) Expression of Wnt1 and $\beta$-catenin was restrained by miR-650 inhibitor in A549 cells. NSCLC, non-small cell lung cancer; ING4, inhibitor of growth $4 .{ }^{* * *} \mathrm{P}<0.01$; ${ }^{* * *} \mathrm{P}<0.001$.

Thus, the association between the expression of ING4 and the 5-year overall survival was detected and it was established that downregulation of ING4 predicted poor prognosis ( $\mathrm{P}=0.0254)$ (Fig. 4B). Moreover, ING4 expression in cells was calculated and the RT-qPCR results indicated that ING4 was downregulated in A549 and NCI-H460 cells versus
MRC-5 ( $\mathrm{P}=0.0008$ and 0.0022) (Fig. 4C). The correlation between miR-650 and ING4 levels and the expression of miR-650 had a negative association with ING4 expression in NSCLC tissues ( $\mathrm{P}=0.0181$ ) (Fig. 4D). The expression of Wnt1 and $\beta$-catenin was restrained by miR-650 inhibitor in A549 cells (Fig. 4E). 

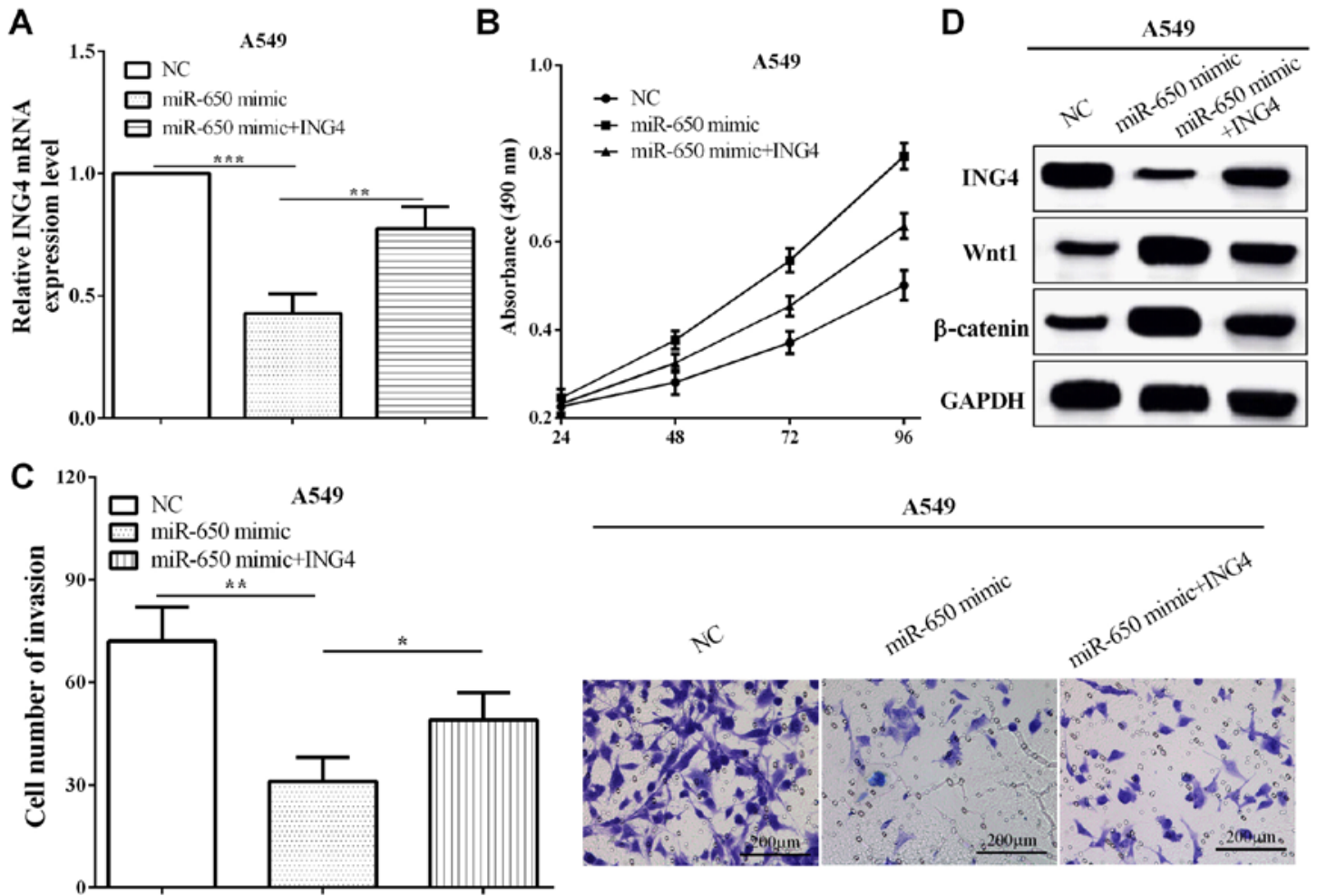

Figure 5. ING4 reverses partial roles of miR-650 on NSCLC cell proliferative and invasive abilities. (A) RT-qPCR effiency of transfecting pcDNA3.1-ING4 plasmid in miR-650 overexpressed A549 cells. (B and C) CCK8 and Transwell assays demonstrated that ING4 suppressed the proliferation and invasion compared with cells only transfected with miR-650 mimic. (D) miR-650 promoted the EMT of NSCLC cells by targeting ING4 through Wnt- $1 / \beta$-catenin pathway. NSCLC, non-small cell lung cancer; ING4, inhibitor of growth 4 ; $\mathrm{NC}$, negative control. ${ }^{*} \mathrm{P}<0.05 ;{ }^{* *} \mathrm{P}<0.01 ;{ }^{* * *} \mathrm{P}<0.001$.

ING4 reverses partial roles of miR-650 on cell proliferative and invasive abilities. To verify the functions of ING4 in miR-650 overexpressed cells, pcDNA3.1-ING4 plasmid was transfected into miR-650 overexpressed A549 cells $(\mathrm{P}=0.0075)$, as shown in Fig. 5A. Subsequently, CCK8 and Transwell assays demonstrated that ING4 suppressed the proliferation and invasion compared with cells only transfected with miR-650 mimic ( $\mathrm{P}=0.0030$ and 0.0427) (Fig. 5B and $\mathrm{C})$. These data demonstrated that ING4 reversed partial roles of miR-650 on NSCLC cell proliferation and invasion. In addition, miR-650 mimic inhibited the expression of ING4, while the expression of Wnt1 and $\beta$-catenin was increased. Re-expression of ING4 reversed the reduction of Wnt1 and $\beta$-catenin in A549 (Fig. 5D). The results indicated that miR-650 promoted NSCLC cell proliferation and invasion by targeting ING4 through Wnt- $1 / \beta$-catenin pathway.

\section{Discussion}

Non-small cell lung cancer, including lung adenocarcinoma, squamous cell carcinoma and large cell carcinoma, has a high morbidity and mortality $(2,27)$. Therefore, it is urgent to explore new biomarkers for disease prevention and clinical treatment of NSCLC.

miRNAs bind to the 3'-UTR of the target mRNA to incise the target mRNAs or to inhibit the translation of proteins at post-transcriptional level (3). miR-650 has been reported to act as a prognostic factor and contributed to the docetaxel chemoresistance in lung adenocarcinoma (28). Our results were consistent with these findings. miR-650 was overexpressed in NSCLC and upregulation of miR-650 predicted poor 5 -year overall survival. Moreover, You et al (29) indicated that miR-650 enhanced cell proliferation, migration and EMT through binding to ING4 in colorectal cancer and Zeng et al (30) in hepatocellular carcinoma. Consistent with all the above findings, we discovered that miR-650 promoted cell proliferation and invasion by targeting ING4 in NSCLC. However, miR-650 suppressed disease progress of rheumatoid arthritis synovial fibroblasts and high-risk non-metastatic colorectal cancer $(31,32)$. Therefore, we considered that the expression of miR-650 had tissue specificity.

ING4 acted as a tumor suppressor, and was a potential target for tumor therapy $(33,34)$. ING4 suppressed cell proliferation and induced cell apoptosis in melanoma (35). Similarly, ING4 inhibited cell growth, invasion of EMT and suppressed tumor angiogenesis (36). Moreover, ING4 suppressed cell proliferation, invasion and enhanced apoptosis in osteosarcoma (37). Our results are consistent with all these findings. ING4 expression was low in NSCLC tissues and cell lines, and downregulation of ING4 predicted poor prognosis. ING4 has been reported to be a target of several miRNAs, including miR-330, miR-423 and miR-761 (38-40). We discovered that ING4 was a direct target of miR-650 and miR-650 mediated the expression of ING4 through directly binding to the 3'-UTR of mRNA. Moreover, miR-650 suppressed Wnt-1/ $\beta$-catenin pathway by targeting ING4 in A549 cells. In addition, ING4 reversed the effects of miR-650 in proliferation and invasion in A549 cells.

In brief, miR-650 was upregulated in NSCLC and upregulation of miR-650 indicated a shorter overall survival of NSCLC, while ING4 demonstrated the opposite results. 
miR-650 promoted cell proliferation and invasion through Wnt- $1 / \beta$-catenin pathway by binding to ING4. ING4 was able to partially reverse the function of miR-650 in cell proliferation and invasion.

\section{Acknowledgements}

Not applicable.

\section{Funding}

No funding was received.

\section{Availability of data and materials}

The datasets used and/or analyzed during the present study are available from the corresponding author on reasonable request.

\section{Authors' contributions}

XT wrote the manuscript. YD performed RT-qPCR and western blot analysis. XiaW and XiuW were responsible for CCK8, transwell and luciferase reporter assays. LZ analyzed and interpreted the patients' data. HB helped with statistical analysis. All authors read and approved the final manuscript.

\section{Ethics approval and consent to participate}

The Ethics Committee of Jining No. 1 People's Hospital (Jining, China) approved this study. Patients who participated in this research had complete clinical data. The signed informed consents were obtained from the patients or the guardians.

\section{Patient consent for publication}

Not applicable.

\section{Competing interests}

The authors declare that they have no competing interests.

\section{References}

1. Siegel RL, Miller KD and Jemal A: Cancer statistics, 2018. CA Cancer J Clin 68: 7-30, 2018.

2. Heist RS and Engelman JA: SnapShot: non-small cell lung cancer. Cancer Cell 21: 448.e2, 2012.

3. Bartel DP: MicroRNAs: Target recognition and regulatory functions. Cell 136: 215-233, 2009.

4. Bartel DP: MicroRNAs: Genomics, biogenesis, mechanism, and function. Cell 116: 281-297, 2004.

5. Garzon R, Calin GA and Croce CM: MicroRNAs in cancer. Annu Rev Med 60: 167-179, 2009.

6. Feng L, Xie Y, Zhang H and Wu Y: Down-regulation of NDRG2 gene expression in human colorectal cancer involves promoter methylation and microRNA-650. Biochem Biophys Res Commun 406: 534-538, 2011.

7. Yang YQ, Tian T, Zhu HY, Liang JH, Wu W, Wu JZ, Xia Y, Wang L, Fan L, Li JY, et al: NDRG2 mRNA levels and miR-28-5p and miR-650 activity in chronic lymphocytic leukemia. BMC Cancer 18: 1009, 2018.

8. Yun JH, Moon S, Lee HS, Hwang MY, Kim YJ, Yu HY, Kim Y, Han BG, Kim BJ and Kim JM: MicroRNA-650 in a copy number-variable region regulates the production of interleukin 6 in human osteosarcoma cells. Oncol Lett 10: 2603-2609, 2015.
9. Sun B, Pu B, Chu D, Chu X, Li W and Wei D: MicroRNA-650 expression in glioma is associated with prognosis of patients. J Neurooncol 115: 375-380, 2013.

10. Mraz M, Dolezalova D, Plevova K, Stano Kozubik K, Mayerova V, Cerna K, Musilova K, Tichy B, Pavlova S, Borsky M, et al: MicroRNA-650 expression is influenced by immunoglobulin gene rearrangement and affects the biology of chronic lymphocytic leukemia. Blood 119: 2110-2113, 2012.

11. Jin $\mathrm{P}$, Chen $\mathrm{H}$, Xie J, Zhou $\mathrm{C}$ and Zhu X: Essential role of microRNA-650 in the regulation of B-cell CLL/lymphoma 11B gene expression following transplantation: A novel mechanism behind the acute rejection of renal allografts. Int J Mol Med 40: 1840-1850, 2017.

12. Ningning S, Libo S, Chuanbin W, Haijiang S and Qing Z: MiR-650 regulates the proliferation, migration and invasion of human oral cancer by targeting growth factor independent 1 (Gfil). Biochimie 156: 69-78, 2019.

13. Yuan C, Xu L, Du P and Pang J: miRNA-650 exerts anti-leukemia activity by inhibiting cell proliferation through Gfil targeting. Tumori 104: 369-374, 2018.

14. Guérillon C, Bigot $\mathrm{N}$ and Pedeux R: The ING tumor suppressor genes: Status in human tumors. Cancer Lett 345: 1-16, 2014.

15. Doyon Y, Cayrou C, Ullah M, Landry AJ, Côté V, Selleck W, Lane WS, Tan S, Yang XJ and Côté J: ING tumor suppressor proteins are critical regulators of chromatin acetylation required for genome expression and perpetuation. Mol Cell 21: 51-64, 2006.

16. Nagashima M, Shiseki M, Pedeux RM, Okamura S, KitahamaShiseki M, Miura K, Yokota J and Harris CC: A novel PHD-finger motif protein, p47ING3, modulates p53-mediated transcription, cell cycle control, and apoptosis. Oncogene 22: 343-350, 2003.

17. Cui S, Gao Y, Zhang K, Chen J, Wang R and Chen L: The emerging role of inhibitor of growth 4 as a tumor suppressor in multiple human cancers. Cell Physiol Biochem 36: 409-422, 2015.

18. Gunduz M, Nagatsuka H, Demircan K, Gunduz E, Cengiz B, Ouchida M, Tsujigiwa H, Yamachika E, Fukushima K, Beder L, et al: Frequent deletion and down-regulation of ING4, a candidate tumor suppressor gene at $12 \mathrm{p} 13$, in head and neck squamous cell carcinomas. Gene 356: 109-117, 2005.

19. Wu Y, Mou X, Wang S, Liu XE and Sun X: ING4 expressing oncolytic vaccinia virus promotes anti-tumor efficiency and synergizes with gemcitabine in pancreatic cancer. Oncotarget 8: 82728-82739, 2017.

20. Keenen MM and Kim S: Tumor suppressor ING4 inhibits estrogen receptor activity in breast cancer cells. Breast Cancer (Dove Med Press) 8: 211-221, 2016.

21. Ren Y, Zhao S, Chen H, Fu YM and Zhao B: Association between the expression of inhibitor of growth family member 4 and the progression of clear cell renal carcinoma. Oncol Lett 14: 2453-2457, 2017.

22. Chen Y, Fu R, Xu M, Huang Y, Sun G and Xu L: N-methylN-nitro-N-nitrosoguanidine-mediated ING4 downregulation contributed to the angiogenesis of transformed human gastric epithelial cells. Life Sci 199: 179-187, 2018.

23. Cai L, Li H, Chen C, Cheng X, Wang Y, Liu J, Wang Y and Hao L: Role of inhibitor of growth 4 in the suppression of human melanoma cells through the Fas/FasL-mediated apoptosis pathway. Int J Mol Med 41: 1055-1061, 2018.

24. Chen Y, Huang Y, Hou P, Zhang Z, Zhang Y, Wang W, Sun G, $\mathrm{Xu} \mathrm{L}$, Zhou J, Bai J, et al: ING4 suppresses tumor angiogenesis and functions as a prognostic marker in human colorectal cancer. Oncotarget 7: 79017-79031, 2016.

25. Pan X, Wang R, Bian H, De W, Zhang P, Wei C and Wang Z: Overexpression of inhibitor of growth 4 enhances radiosensitivity in non-small cell lung cancer cell line SPC-A1. Technol Cancer Res Treat 16: 533-545, 2017.

26. Livak KJ and Schmittgen TD: Analysis of relative gene expression data using real-time quantitative PCR and the 2(-Delta Delta C(T)) method. Methods 25: 402-408, 2001.

27. Ettinger DS, Akerley W, Borghaei H, Chang AC, Cheney RT, Chirieac LR, D'Amico TA, Demmy TL, Govindan R, Grannis FW Jr, et al; National comprehensive cancer network: Non-small cell lung cancer, version 2.2013. J Natl Compr Canc Netw 11: 645-653, quiz 653, 2013.

28. Huang JY, Cui SY, Chen YT, Song HZ, Huang GC, Feng B, Sun M, De W, Wang R and Chen LB: MicroRNA-650 was a prognostic factor in human lung adenocarcinoma and confers the docetaxel chemoresistance of lung adenocarcinoma cells via regulating Bcl-2/Bax expression. PLoS One 8: e72615, 2013. 
29. You Q, Li H, Liu Y, Xu Y, Miao S, Yao G, Xue Y, Geng J, Jin X and Meng H: MicroRNA-650 targets inhibitor of growth 4 to promote colorectal cancer progression via mitogen activated protein kinase signaling. Oncol Lett 16: 2326-2334, 2018.

30. Zeng ZL, Li FJ, Gao F, Sun DS and Yao L: Upregulation of miR-650 is correlated with down-regulation of ING4 and progression of hepatocellular carcinoma. J Surg Oncol 107: $105-110,2013$

31. Xu X, Chen H, Zhang Q, Xu J, Shi Q and Wang M: MiR-650 inhibits proliferation, migration and invasion of rheumatoid arthritis synovial fibroblasts by targeting AKT2. Biomed Pharmacother 88: 535-541, 2017.

32. Zhou C, Cui F, Li J, Wang D, Wei Y, Wu Y, Wang J, Zhu H and Wang S: MiR-650 represses high-risk non-metastatic colorectal cancer progression via inhibition of AKT2/GSK3 $\beta /$ E-cadherin pathway. Oncotarget 8: 49534-49547, 2017.

33. Li S, Fan T, Liu H, Chen J, Qin C and Ren X: Tumor suppressor ING4 overexpression contributes to proliferation and invasion inhibition in gastric carcinoma by suppressing the NF- $\kappa \mathrm{B}$ signaling pathway. Mol Biol Rep 40: 5723-5732, 2013.

34. Yuan S, Jin J, Shi J and Hou Y: Inhibitor of growth-4 is a potential target for cancer therapy. Tumour Biol 37: 4275-4279, 2016.

35. Ma Y, Cheng X, Wang F, Pan J, Liu J, Chen H, Wang Y and Cai L: ING4 inhibits proliferation and induces apoptosis in human melanoma A375 cells via the Fas/caspase- 8 apoptosis pathway. Dermatology 232: 265-272, 2016.
36. Qu H, Yin H, Yan S, Tao M, Xie Y and Chen W: Inhibitor of growth 4 suppresses colorectal cancer growth and invasion by inducing G1 arrest, inhibiting tumor angiogenesis and reversing epithelial-mesenchymal transition. Oncol Rep 35: 2927-2935, 2016.

37. Li M, Zhu Y, Zhang H, Li L, He P, Xia H, Zhang Y and Mao C: Delivery of inhibitor of growth 4 (ING4) gene significantly inhibits proliferation and invasion and promotes apoptosis of human osteosarcoma cells. Sci Rep 4: 7380, 2014.

38. Hu X, Feng Y, Sun L, Qu L and Sun C: Roles of microRNA-330 and its target gene ING4 in the development of aggressive phenotype in hepatocellular carcinoma cells. Dig Dis Sci 62: 715-722, 2017.

39. Li S, Zeng A, Hu Q, Yan W, Liu Y and You Y: miR-423-5p contributes to a malignant phenotype and temozolomide chemoresistance in glioblastomas. Neuro Oncol 19: 55-65, 2017.

40. Yan A, Yang C, Chen Z, Li C and Cai L: MiR-761 promotes progression and metastasis of non-small cell lung cancer by targeting ING4 and TIMP2. Cell Physiol Biochem 37: 55-66, 2015.

This work is licensed under a Creative Commons Attribution-NonCommercial-NoDerivatives 4.0 International (CC BY-NC-ND 4.0) License. 